\title{
Influência de diferentes solventes na síntese de mulita por sol-gel
}

\author{
Influence of different solvents in the \\ synthesis of mullite by sol-gel
}

\begin{abstract}
Aluska do Nascimento Simões Braga ${ }^{1,2}$, Geysivana Kessya Garcia Carvalho ${ }^{1}$, Hélio de Lucena Lira ${ }^{3}$, Gelmires de Araújo Neves ${ }^{3}$, Romualdo Rodrigues Menezes ${ }^{3}$
\end{abstract}

\footnotetext{
${ }^{1}$ Coordenação de Engenharia de Materiais, CT/UFPI, Av. Universitária, s/n, Teresina, PI, Brasil

${ }^{2}$ Programa de pós-Graduação em Engenharia de Materiais, PPGEM/IFPI, Rua Álvaro Mendes, 94, Teresina, PI, Brasil

${ }^{3}$ Programa de Pós-Graduação em Ciência e Engenharia de Materiais, PPG-CEMAT/UFCG, Rua. Aprígio Veloso, 882, Campina Grande, PB, Brasil e-mail: aluskasimoes@hotmail.com, Geysivanacarvalho@hotmail.com, helio.lira@ufcg.edu.br gelmiresneves@gmail.com,romualdomenezes@gmail.com
}

\section{RESUMO}

Mulita é um material que tem recebido atenção especial devido às suas propriedades químicas, físicas e mecânicas, que estão correlacionadas com o método de síntese utilizado. O método sol-gel destaca-se frente aos outros métodos químicos devido à possibilidade de obtenção de materiais com elevado grau de pureza e baixas temperaturas de síntese. Sendo assim, este trabalho teve por objetivo a análise dos solventes água, álcool etílico, álcool isopropílico e tetrahidrofurano, na obtenção de mulita por meio da rota sol-gel. As amostras foram caracterizadas por difração de raios X (DRX), análise termogravimétrica e diferencial (TG/ATD), adsorção de nitrogênio, microscopia eletrônica de varredura (MEV) e microscopia eletrônica de transmissão (MET). Os resultados mostraram que a mulita foi sintetizada com todos os solventes, porém, o uso dos álcoois como solvente proporcionou a obtenção das amostras mais cristalinas, com as menores perdas de massa, com uma morfologia formada por agregados e partículas individualizadas.

Palavras-chave: Síntese. Sol-gel. Mulita. Solventes.

\begin{abstract}
Mullite is a material that has received special attention due to its chemical, physical and mechanical properties, which are correlated with the synthesis method used. The sol-gel method stands out compared to other chemical methods due to the possibility of obtaining materials with a high degree of purity and low synthesis temperatures. Thus, this work aimed to analyze the solvents water, ethyl alcohol, isopropyl alcohol and tetrahydrofuran, in obtaining mullite using the sol-gel route. The samples were characterized by X-ray diffraction (XRD), thermogravimetric and differential analysis (TG/DTA), nitrogen adsorption (BET), scanning electron microscopy (SEM) and transmission electron microscopy (MET). The results showed that mullite was synthesized with all solvents, however, the use of alcohols as a solvent provided the obtaining of the most crystalline samples, with the lowest mass losses, with a morphology formed by aggregates and individualized particles.
\end{abstract}

Keywords: Synthesis. Sol-gel. Mullite. Solvents. 


\section{INTRODUÇÃO}

A Mulita é um aluminossilicato que alcançou extensa importância devido às suas excelentes propriedades [14], tendo uma vasta gama de aplicações, como por exemplo: membranas para separação de gases, reforço mecânico em matriz cerâmica, para tratamento de água, em radomes, etc [5-10]. Assim, estudos voltados para a síntese de mulita têm uma grande importância científica e tecnológica, sendo presente em vários laboratórios de cerâmica em todo o mundo.

A síntese convencional de mulita requer temperaturas muito elevadas $\left(1600-1700^{\circ} \mathrm{C}\right)$, os tempos de reação são longos e o produto final geralmente tem composições variáveis [11-13]. A fim de minimizar estes problemas vários métodos químicos de síntese de mulita foram desenvolvidos [14-20]. Dentre esses métodos, destaca-se o método sol-gel, que é o método químico mais utilizado na síntese de mulita. Sendo um método versátil na síntese de materiais inorgânicos, que possui a vantagem de fornecer materiais com elevada pureza e homogeneidade química, além de boa reprodutibilidade [21-24]. A versatilidade deste método é uma de suas maiores vantagens, podendo obter os materiais através de diferentes soluções, sejam elas coloidais ou poliméricas, ou através de variações do método [25-27].

Sabe-se que na tecnologia sol-gel a escolha das variáveis de síntese afeta diretamente as propriedades do produto final obtido e que o tipo de solvente é um fator de grande importância, interferindo significativamente na homogeneidade química do sistema. A síntese através do método sol-gel pode ser realizada com uma ampla gama de diferentes solventes onde eles também podem influenciar o estágio inicial do equilíbrio químico. Diferentes moléculas de solvente, tendo massas molares diferentes, comprimentos de cadeia, momentos dipolares, constantes dielétricas, podem modular reações de complexação, processo de condensação e afetar a extensão de interpenetração do gel. Os solventes podem, por viscosidade, influenciar as capacidades de migração das espécies, como também podem reagir com os precursores e, assim, alterá-los no nível molecular, alterando todo o processo sol-gel [28]. Várias pesquisas relatam a importância da escolha do solvente nas características finais dos materiais sintetizados [16, 28-30].

Assim, diante da relevância do método sol-gel na obtenção da fase mulita, percebe-se a necessidade de uma maior quantidade de pesquisas abordando a influência de diversos solventes, de modo a melhor compreender o efeito desses nas características finais da mulita. Deste modo, o presente trabalho teve como objetivo estudar a influência de diferentes solventes na síntese de mulita através da rota sol-gel.

\section{MATERIAIS E MÉTODOS}

A mulita foi preparada pelo método sol-gel utilizando como precursores nitrato de alumínio nano-hidratado (Neon, 98\%) e tetraetilortossilicato (TEOS, Fluka, 99\%). Os precursores foram utilizados de modo a se obter uma proporção de Al:Si de 3:1. Foram utilizados como solventes: álcool etílico (Santa Cruz, PA); álcool isopropílico (Cinética, PA); tetrahidrofurano (THF, Nuclear, 99\%); água com $\mathrm{pH}$ ácido ( $\mathrm{pH} \sim 2$, corrigido com $\mathrm{HCl}$, Fmaia) e água com $\mathrm{pH}$ básico ( $\mathrm{pH} \sim 12$, corrigido com $\mathrm{NH}_{4} \mathrm{OH}$, Vetec). Inicialmente, 100ml de cada solvente em estudo foi aquecido a uma temperatura de $60^{\circ} \mathrm{C}$, em seguida, foram adicionados $19,51 \mathrm{ml} \mathrm{de}$ TEOS e 105,52g de nitrato de alumínio. Esse sistema foi mantido sob refluxo durante 5 dias com agitação constante e temperatura controlada em $60^{\circ} \mathrm{C}$. Após os 5 dias, a solução obtida foi mantida em estufa por mais 4 dias, também com temperatura controlada em $60^{\circ} \mathrm{C}$, formando um gel. Os géis obtidos foram calcinados a $450^{\circ} \mathrm{C}$ por 2 horas, com taxa de aquecimento de $5^{\circ} \mathrm{C} / \mathrm{min}$, para eliminação da matéria orgânica. Em seguida, os pós obtidos foram desaglomerados em um almofariz, passados em peneira ABNT ${ }^{\circ} 200(0,074 \mathrm{~mm})$, e calcinados nas temperaturas de $1100^{\circ} \mathrm{C}$ e $1200^{\circ} \mathrm{C}$, durante 2 horas, com taxa de aquecimento de $5^{\circ} \mathrm{C} / \mathrm{min}$, para obtenção da fase mulita. As amostras foram denominadas de Etil, Iso, THF, AC e AB quando foram usados os solventes álcool etílico, álcool isopropílico, THF, água acidificada e água basificada, respectivamente.

Os materiais foram analisados por difração de raios X (Shimadzu, XRD-6000, CuKa) para identificação das fases presentes e grau de cristalinidade. Na identificação das amostras foram utilizadas fichas do Joint Committee on Powder Diffraction Standards (JCPDS), banco de dados PCPDFWIN: JCPDS 15-0776 para mulita, e JCPDS 37-1483 para os picos de espinélio. A cristalinidade foi determinada a partir da razão entre a área integrada do pico referente à fase cristalina e a área referente à fração amorfa (Shimadzu, software Search Match). Os materiais foram caracterizados também por: análise termogravimétrica e diferencial (TG/ATD, Shimadzu, TA-60), em atmosfera de ar e taxa de aquecimento de $10^{\circ} \mathrm{C} / \mathrm{min}$; determinação da área superficial por adsorção de $\mathrm{N}_{2}$ (Micromeritics, ASAP 2020); análise dos aspectos morfológicos por microscopia eletrônica de varredura (MEV, Philips, XL-30 FEG); e microscopia eletrônica de transmissão (MET, Philips, CM-120). 


\section{RESULTADOS E DISCUSSÃO}

As Figuras. 1a e 1b ilustram os difratogramas de raios $\mathrm{X}$ das amostras Etil, Iso, THF, AC, AB, que foram sintetizadas utilizando como solventes o álcool etílico, álcool isopropílico, THF, água acidificada e água basificada, calcinadas em $1100^{\circ} \mathrm{C} / 2 \mathrm{~h}$ e $1200^{\circ} \mathrm{C} / 2 \mathrm{~h}$, respectivamente.

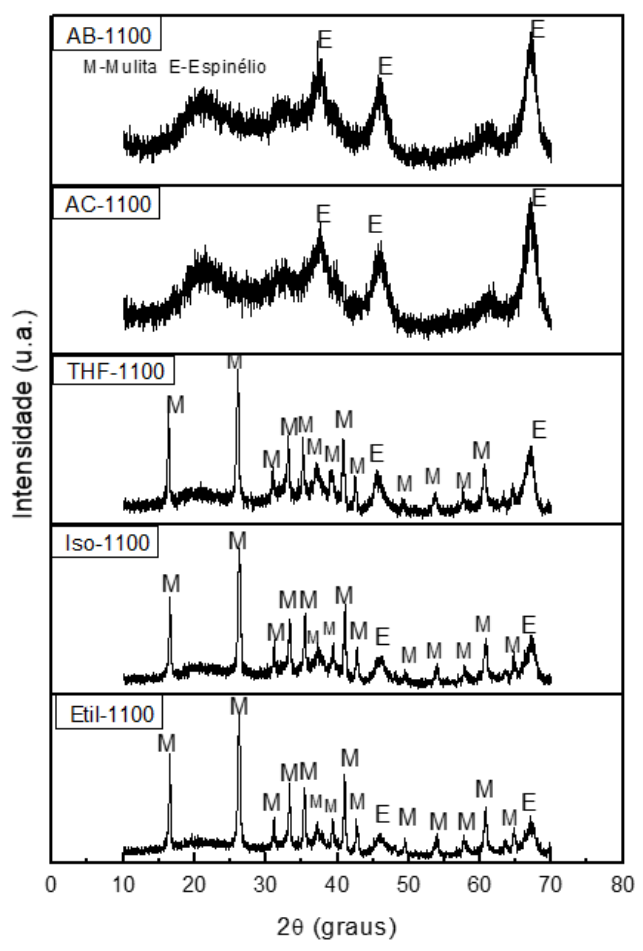

(a)

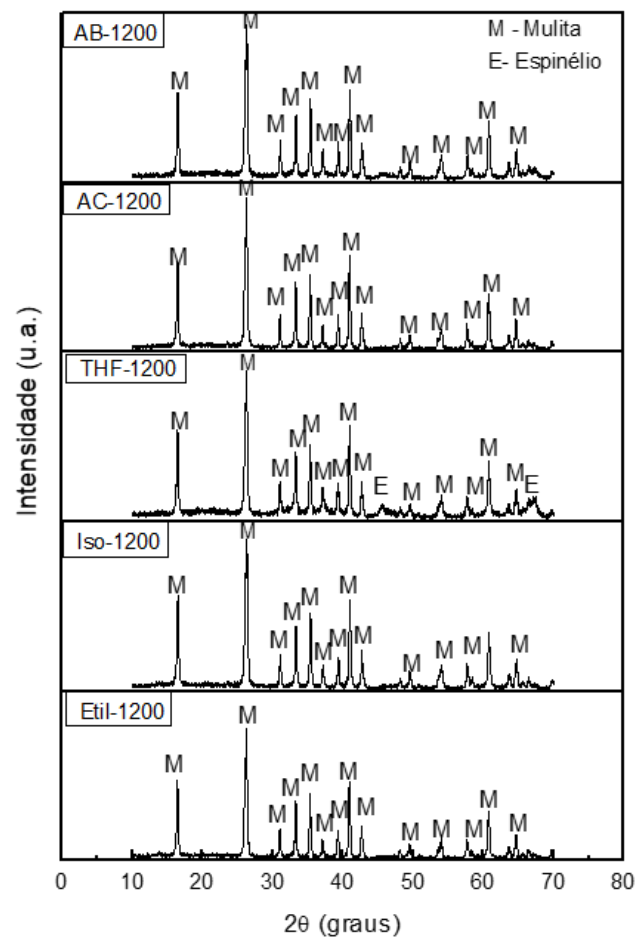

(b)

Figura 1: Difratogramas de raios $\mathrm{X}$ das amostras Etil, Iso, THF, $\mathrm{AC}, \mathrm{AB}$, que foram sintetizadas utilizando como solventes o álcool etílico, álcool isopropílico, THF, água acidificada e água basificada, calcinadas em $1100^{\circ} \mathrm{C} / 2 \mathrm{~h}$ (a) e $1200^{\circ} \mathrm{C} / 2 \mathrm{~h}(\mathrm{~b})$, respectivamente.

Analisando os difratogramas da Figura 1, quando se utiliza os álcoois etílico e isopropílico como solventes (Amostras Etil e Iso) percebe-se a cristalização da mulita já na temperatura de $1100^{\circ} \mathrm{C}$, com discreta formação de espinélio. Ao aumentar a temperatura para $1200^{\circ} \mathrm{C}$ a fase mulita formada apresenta um padrão mais cristalino, e sem a formação de espinélio, constatando alta homogeneidade do sistema.

Outros pesquisadores [1] também prepararam géis de mulita sob condições de refluxo durante 7 dias, também usando o álcool etílico, o nitrato de alumínio e o TEOS, como reagentes. A formação da fase mulita é observada em baixas temperaturas, $900^{\circ} \mathrm{C}$, porém elevados tempos de envelhecimento ( 1 mês) foram necessários. Outras pesquisas [32-35] sintetizando mulita em condições de síntese semelhantes ao desta pesquisa, destacaram a obtenção de mulita em faixa de temperaturas correspondente ao deste trabalho.

Quando o THF é usado como solvente também se observa a cristalização da mulita, juntamente com uma discreta formação de espinélio, para ambas as temperaturas de calcinação. Comparando os padrões de raios $\mathrm{X}$ quando do uso dos álcoois e do THF, percebe-se que as amostras sintetizadas com os álcoois são mais cristalinas. Acredita-se que isso possa estar relacionado a menor capacidade de dissolução do THF, devido a seu baixo valor de constante dielétrica e ao fato do THF possuir alta volatilidade. Essas características podem fazer com que ocorra a formação de núcleos rapidamente, mas fora da composição estequiométrica da mulita, o que provocou a formação de espinélio.

Analisando os difratogramas das amostras quando se utiliza a água acidificada (amostra AC) como solvente, percebe-se que na temperatura de $1100^{\circ} \mathrm{C}$ há bandas de material amorfo e reflexões relacionadas a fase espinélio, com baixa cristalinidade. Com a elevação da temperatura para $1200^{\circ} \mathrm{C}$ a fase mulita é cristalizada, sem a formação de espinélio, e com elevada cristalinidade. Nesta reação, o objetivo era reduzir os pro- 
blemas do uso da água como solvente, como relatado na literatura [16], e que estes fossem suprimidos pelo $\mathrm{pH}$, como evidenciado por outras pesquisas [36]. Isso por que em condições ácidas, o grupamento silanol $(\mathrm{SiOH})$ que é o resultado da hidrólise do TEOS, sofre desidratação dos íons $\mathrm{OH}^{-}\left(\mathrm{SiOH}+\mathrm{H}^{+} \rightarrow \mathrm{Si}^{+}+\mathrm{H}_{2} \mathrm{O}\right)$ diminuindo a aglomeração das partículas de sílica. Dessa forma as ligações Si-O-Al são facilitadas, formando a mulita.

Quando se utiliza a água basificada (amostra $\mathrm{AB}$ ) como solvente os padrões são semelhantes aos da água acidificada, ou seja, em $1100^{\circ} \mathrm{C}$ a amostra ainda é praticamente amorfa, e com a elevação da temperatura para $1200^{\circ} \mathrm{C}$ a mulita é formada, sem a presença de espinélio, com aumento da cristalinidade. Estes resultados são explicados pelo fato do grupamento silanol ser anfótero, isto é, sofre tanto protonação em meio ácido, formando complexos catiônicos (positivos), quanto desprotonação em meio básico, formando complexos aniônicos. Então, nesta reação onde a água basificada foi usada, os grupos hidroxilas reagiram facilmente com os silanois $\left(\mathrm{SiOH}+\mathrm{OH}^{-} \rightarrow \mathrm{SiO}^{-}+\mathrm{H}_{2} \mathrm{O}\right)$, promovendo a dissolução da sílica, facilitando também as ligações Si-O-Al, e formando a mulita.

A Tabela 1 apresenta os valores de cristalinidade das amostras Etil, Iso, THF, AC, AB, que foram sintetizadas utilizando como solventes o álcool etílico, álcool isopropilico, THF, água acidificada e água basificada, calcinadas em $1100^{\circ} \mathrm{C}$ e $1200^{\circ} \mathrm{C}$. Nota-se que os melhores valores de cristalinidade foram obtidos nas amostras sintetizadas com os álcoois, em ambas as temperaturas de calcinação, como também constatado pelos difratogramas.

Tabela 1: Cristalinidade das amostras Etil, Iso, THF, AC, AB, que foram sintetizadas utilizando como solventes o álcool etílico, álcool isopropilico, THF, água acidificada e água basificada, calcinadas em $1100^{\circ} \mathrm{C}$ e $1200^{\circ} \mathrm{C}$.

\begin{tabular}{ccc}
\hline AMOSTRAS & \multicolumn{3}{c}{ CRISTALINIDADE (\%) } \\
\cline { 2 - 3 } & $\mathbf{1 1 0 0 ^ { \circ } \mathbf { C }}$ & $\mathbf{1 2 0 0 ^ { \circ } \mathbf { C }}$ \\
\hline Etil & 58,2 & 91,7 \\
Iso & 52,3 & 90,2 \\
THF & 47,5 & 73,7 \\
AC & 31,2 & 87,6 \\
AB & 32,6 & 85,2 \\
\hline
\end{tabular}

A Figura 2 apresenta as curvas de TG/ATD das amostras a) Etil, b) Iso, c) THF, d) AC e d) AB, que foram sintetizadas utilizando como solventes o álcool etílico, álcool isopropílico, THF, água acidificada e água basificada, respectivamente. 

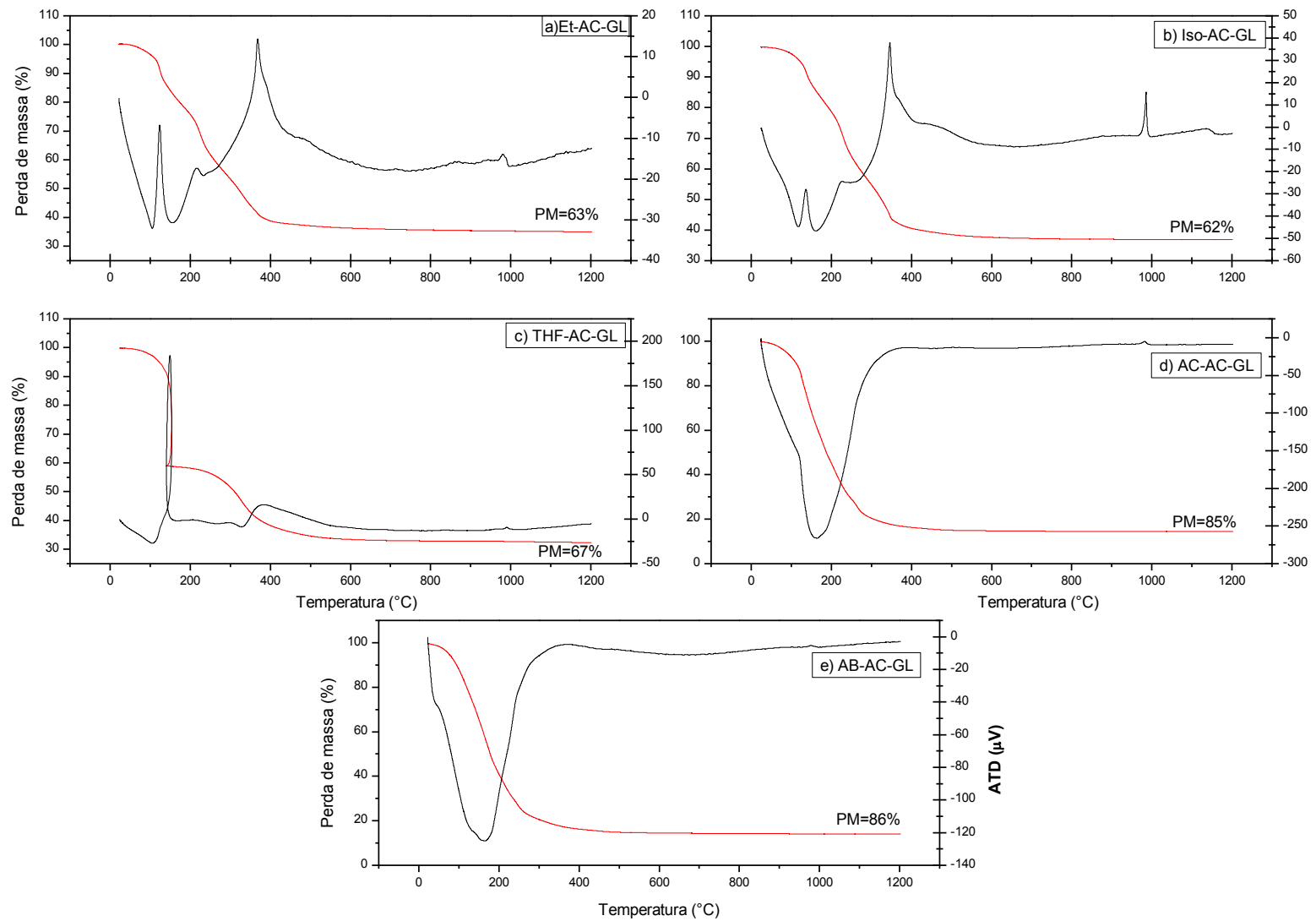

Figura 2: Curvas de TG/ATD das amostras a) Etil, b) Iso, c) THF, d) AC e e) AB, que foram sintetizadas utilizando como solventes o álcool etílico, álcool isopropílico, THF, água acidificada e água basificada.

Analisando as curvas de TG/ATD das amostras sintetizadas com os álcoois etílico e isopropílico, percebe-se uma similaridade, onde o processo de decomposição ocorreu em faixas de temperaturas semelhantes. Para essas amostras (Etil e Iso) a decomposição ocorreu em duas etapas. A primeira iniciou em aproximadamente $50^{\circ} \mathrm{C}$ até $200^{\circ} \mathrm{C}$, correspondendo a perda de água e do álcool. A segunda etapa que ocorreu em aproximadamente $200^{\circ} \mathrm{C}$ até $400^{\circ} \mathrm{C}$, é referente a combustão de matéria orgânica e da decomposição dos precursores metálicos (TEOS e nitrato). As amostras apresentaram perdas de massa de aproximadamente $60 \%$. As curvas de ATD dessas amostras apresentaram três picos endotérmicos em aproximadamente 100, 150 e $230^{\circ} \mathrm{C}$, referentes às perdas de massa. Com uma banda exotérmica em aproximadamente $370^{\circ} \mathrm{C}$, referente à formação dos óxidos de alumínio e de silício. Apresentaram também um pequeno pico exotérmico em aproximadamente $980^{\circ} \mathrm{C}$, que corresponde ao início da cristalização da mulita. Apenas as amostras sintetizadas com ambos os álcoois apresentaram o pico exotérmico em $980^{\circ} \mathrm{C}$, devido a maior cristalinidade dessas amostras, como evidenciado nos difratogramas de raios $\mathrm{X}$.

Para a amostra THF, a primeira etapa ocorre no intervalo de $50^{\circ} \mathrm{C}$ até $140^{\circ} \mathrm{C}$, e a segunda etapa no intervalo de $140^{\circ} \mathrm{C}$ até $500^{\circ} \mathrm{C}$. Ambas as etapas correspondem às mesmas perdas que ocorreram para as amostras Etil e Iso. Porém, percebe-se que a primeira etapa de decomposição desta amostra ocorre mais rapidamente, comparada com as demais amostras, isso é indicativo da cinética do processo de decomposição térmica, que na amostra THF ocorreu de forma bem mais rápida, devido à baixa temperatura de evaporação do solvente THF. A perda de massa dessa amostra foi de 67\%. A curva de ATD da amostra THF apresenta um pico exotérmico em aproximadamente $140^{\circ} \mathrm{C}$. Esse pico é referente a formação da rede de ligações $\mathrm{Si}$-O-Si e Al-O-Al, devido a rápida liberação do solvente THF. Esse comportamento também foi relatado em outro estudo sobre a síntese de mulita [37], onde a perda do solvente ocasionou as reações das espécies, formando a rede de ligações $\mathrm{Si}-\mathrm{O}-\mathrm{Al}$, em baixas temperaturas, fornecendo liberação de calor, interpretado por um pico 
exotérmico. A amostra THF também apresentou um ombro exotérmico, em aproximadamente $400^{\circ} \mathrm{C}$, atribuído a formação dos óxidos.

As amostras $\mathrm{AC}$ e $\mathrm{AB}$, que usaram água acidificada e basificada como solventes, respectivamente, apresentam uma maior similaridade, com perdas de massa na mesma faixa de temperatura, e em uma única etapa. Iniciando em $60^{\circ} \mathrm{C}$ até $500^{\circ} \mathrm{C}$, referentes às mesmas perdas que ocorreu nas amostras anteriores. Essas amostras apresentam perdas de massa de $85 \%$. Ocorreu essa maior perda de massa, comparada com as amostras Etil, Iso e THF, pois como o solvente foi a água, mais grupos silanóis foram formados, devido a maior taxa de hidrólise da sílica, e com isso uma maior desidratação ocorreu. As curvas de ATD das amostras AC e $\mathrm{AB}$ apresentaram um pico endotérmico em $160^{\circ} \mathrm{C}$, associado as perdas de massa.

A Tabela 2 apresenta os valores da caracterização textural das amostras Etil, Iso, THF, AC e AB, sintetizadas utilizando como solventes o álcool etílico, álcool isopropílico, THF, água acidificada e água basificada, calcinadas a $1200^{\circ} \mathrm{C}$, respectivamente. Onde $\mathrm{S}_{\mathrm{BET}}$ corresponde ao valor da área superficial determinada pelo método BET e Vp ao volume de poro e Dp ao diâmetro médio de poros, calculados pelo método BJH.

Tabela 2: Valores de área superficial $\left(\mathrm{S}_{\mathrm{BET}}\right)$, volume de poro $(\mathrm{Vp})$ e diâmetro médio de poro $(\mathrm{Dp})$ das amostras Etil, Iso, $\mathrm{THF}, \mathrm{AC}$ e $\mathrm{AB}$, calcinadas a $1200^{\circ} \mathrm{C}$.

\begin{tabular}{cccc}
\hline AMOSTRA & $\mathbf{S}_{\mathrm{BET}}\left(\mathrm{m}^{2} / \mathbf{g}\right)$ & $\mathbf{V p}\left(\mathbf{c m}^{3} / \mathbf{g}\right)$ & $\mathbf{D p}(\dot{\mathbf{A}})$ \\
\hline Etil & 2,8 & 0,0138 & 211 \\
Iso & 4,8 & 0,0162 & 173 \\
THF & 1,5 & 0,0052 & 217 \\
AC & 4,8 & 0,0158 & 138 \\
AB & 7,5 & 0,0174 & 99 \\
\hline
\end{tabular}

Para todas as amostras, os dados contidos na Tabela 2 não apresentaram variações significativas, onde os valores de área superficial estão em acordo com dados da literatura, para amostras sintetizadas por outras rotas sol-gel [ $\underline{38}, \underline{39}]$. Os valores médios de diâmetro dos poros são todos de dimensões mesoporosas.

A amostra sintetizada com o THF é a que apresentou o menor valor de área superficial. Possivelmente, isso ocorreu devido a maior quantidade de fase amorfa presente nesta amostra, que transformou-se em fase vítrea durante o aquecimento, ou com comportamento viscoelástico, suficiente para agregar as partículas menores, resultando na diminuição da área. Isto também pode ser constatado pela diminuição do volume total de poros desta amostra, o que implica em um maior processo de densificação, causado pela fase vítrea.

As amostras sintetizadas usando água como solvente (tanto em meio ácido como em meio básico) apresentaram os maiores valores de área superficial. Provavelmente, por uma menor agregação das partículas durante a queima, que pode ter ocorrido por apresentarem tamanhos de partículas maiores que as obtidas com os outros solventes; ou porque a fase amorfa presente não resultou em fase vítrea após queima (fase amorfa com elevado teor de alumina).

A Figura 3 (a-e) exibe a morfologia obtida por microscopia eletrônica de varredura das amostras Etil, Iso, THF, $\mathrm{AC}$ e $\mathrm{AB}$, sintetizadas utilizando como solventes o álcool etílico, álcool isopropílico, THF, água acidificada e água basificada, calcinadas a $1200^{\circ} \mathrm{C}$, respectivamente. 

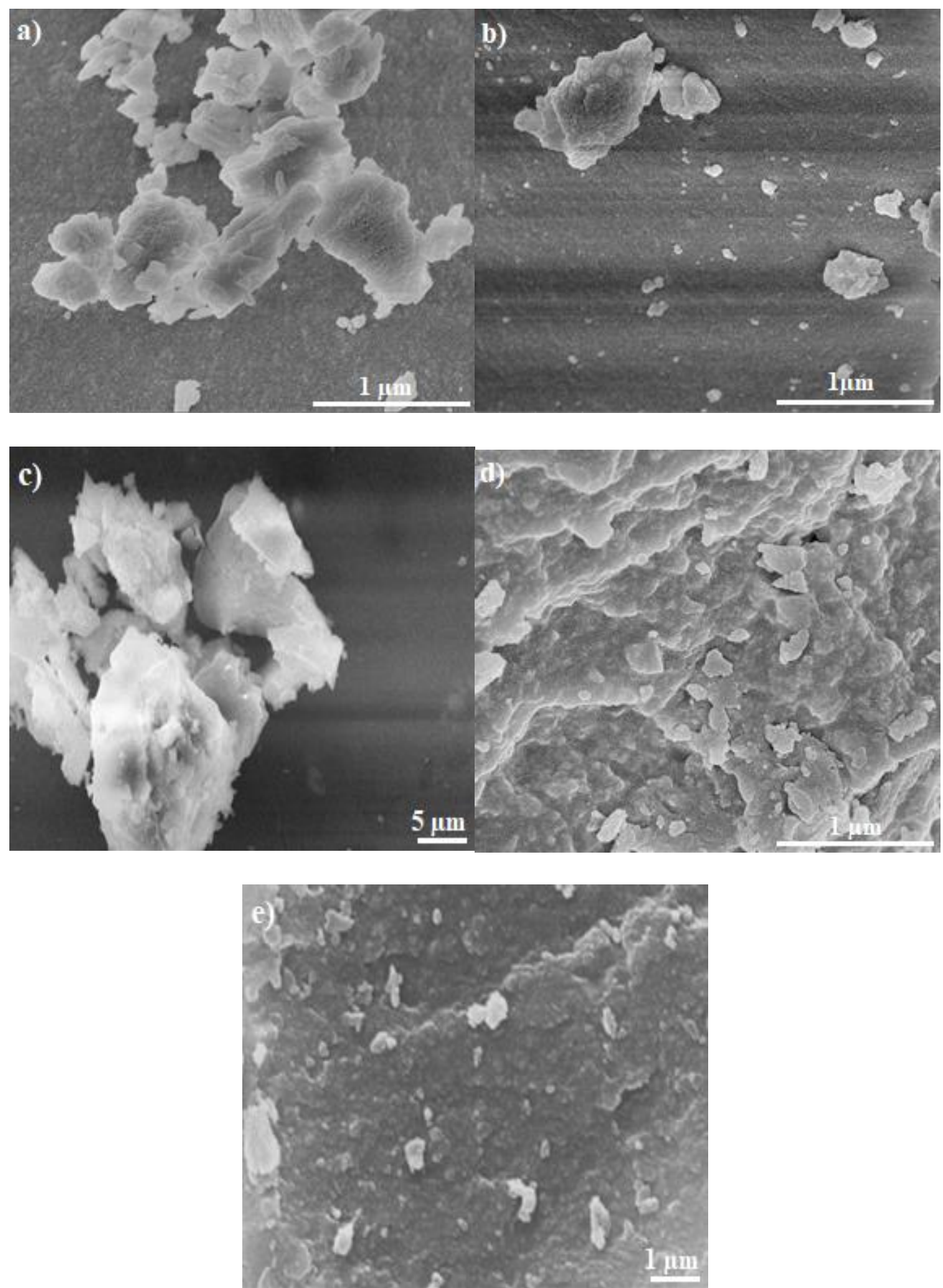

Figura 3: Morfologia obtida por microscopia eletrônica de varredura das amostras a) Etil, b) Iso, c) THF, d) AC e e) AB, sintetizadas utilizando como solventes o álcool etílico, álcool isopropílico, THF, água acidificada e água basificada, calcinadas a $1200^{\circ} \mathrm{C}$, respectivamente.

Analisando as micrografias da Figura 3, percebe-se que as amostras sintetizadas com os álcoois etílico (a) e isopropílico (b) possuem morfologias semelhantes, formadas por agregados e também se observa algumas partículas individualizadas. Os agregados possuem formato irregular e são constituídos de partículas nanométricas, também com formato irregular, com dimensões da ordem de 100 a 500nm. 
Quando se usa o THF (c) como solvente, os agregados formados são irregulares possuindo um tamanho aparentemente maior, comparado com os aglomerados obtidos quando se usa os álcoois, e não se observa as partículas individualizadas, corroborando com os dados obtidos por adsorção de nitrogênio. Provavelmente as heterogeneidades que ocorrem no gel quando do uso do THF, favorece a segregação das fases, por isso as partículas individualizadas não são observadas. De acordo com os resultados de difração de raios $\mathrm{X}$ a amostra sintetizada com THF é menos cristalina comparada com as amostras sintetizadas com os álcoois, dessa forma, a formação de uma fase vítrea possivelmente também ajudou a agregar as partículas por capilaridade.

Ao se usar a água acidificada (d) e basificada (e) como solvente a morfologia das amostras é semelhante, possuindo agregados de formato irregular e os grãos observados possuem morfologia esférica. No entanto, o tamanho dos agregados é maior que no caso das amostras sintetizadas usando álcool etílico e isopropílico. Ambas as amostras, acidificada e basificada, apresentam aparentemente a presença de uma fase vítrea, resultando em um aspecto de maior densificação, provocado pelo maior tamanho do agregado que não pode ser observado individualizado. De acordo com a literatura [40] essa densificação é consequência do uso de elevado teor de água que promove a reação de hidrólise, atingindo um maior grau de condensação.

As Figuras 4(a) e 4(b) exibem a micrografia eletrônica de transmissão e o padrão de difração de elétrons, respectivamente, da amostra Etil. Enquanto as Figuras 4(c) e 4(d) exibem a micrografia eletrônica de transmissão e o padrão de difração de elétrons, respectivamente, da amostra Iso. Ambas calcinadas a $1200^{\circ} \mathrm{C}$.
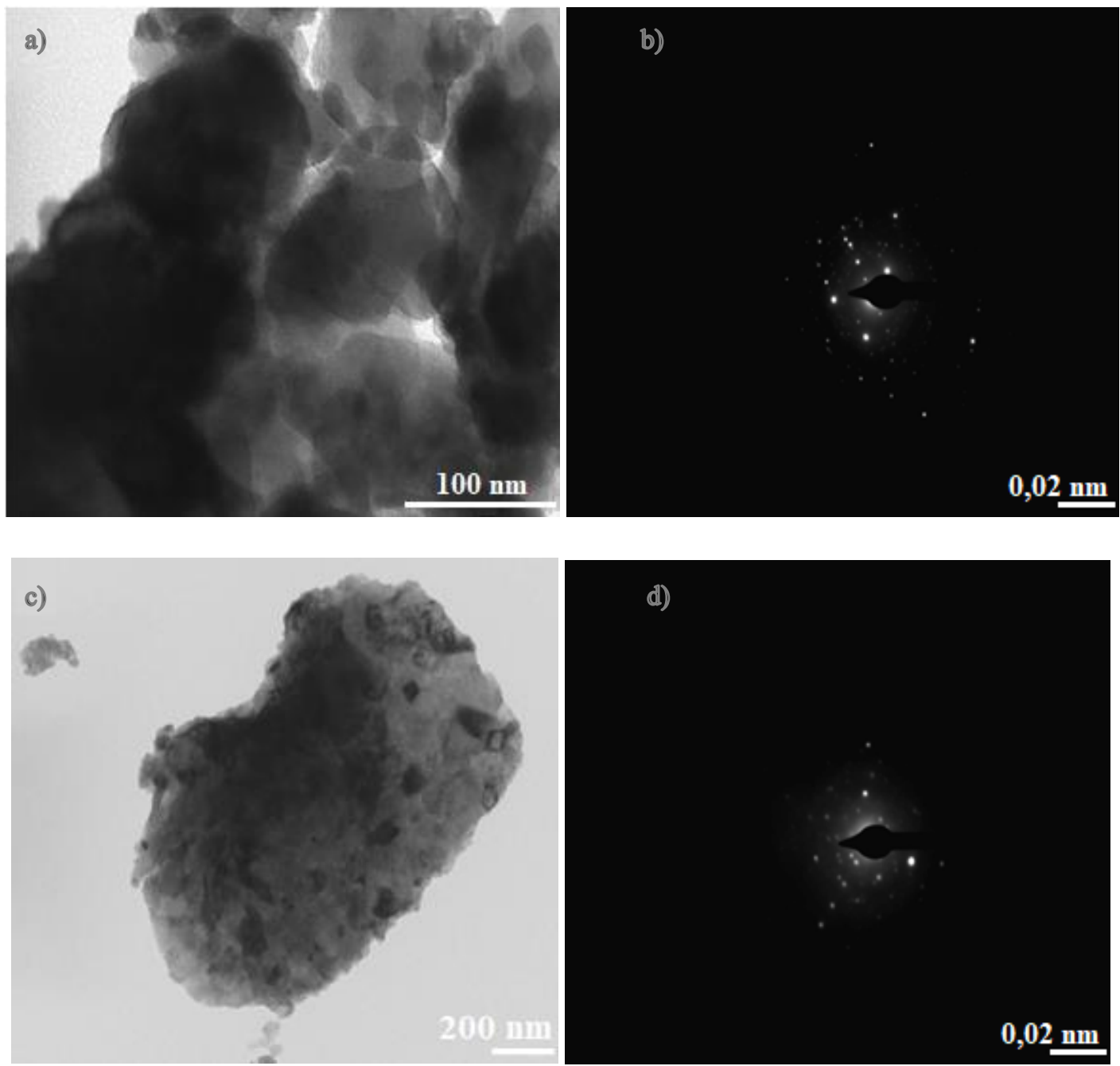

Figura 4: Microscopia eletrônica de transmissão das amostras a) Etil e c) Iso, e os respectivos padrões de difração das amostras b) Etil e d) Iso, obtidas utilizando álcool etílico e isopropílico como solventes, calcinadas a $1200^{\circ} \mathrm{C}$, respectivamente.

Analisando as micrografias da Figura 4, percebe-se que ambas as amostras apresentam um perfil semelhante, formadas por partículas primárias, da ordem de $100 \mathrm{~nm}$, que aglomeraram, formando partículas 
secundárias, relativamente compactas, e de formato irregular. Comparando os padrões de difração de elétrons, nota-se que a amostra sintetizada com álcool etílico apresentou maior cristalinidade, possuindo mais pontos de difração, relacionados com a fase mulita, enquanto a amostra sintetizada com álcool isopropílico apresentou uma maior quantidade de halos difusos, referente a fase amorfa, corroborando com os resultados da difração de raios X.

\section{CONCLUSÕES}

O método sol-gel é eficaz na obtenção de mulita com boa homogeneidade química. Todo os solventes estudados cristalizaram mulita, porém, o tipo de solvente teve influência nas características estruturas, textuais e morfológicas das amostras obtidas. De todos os solventes estudados, os álcoois, etílico e isopropílico, foram os melhores solventes para síntese de mulita, formando a fase em temperaturas mais baixas e com maior grau de cristalinidade, menores perdas de massa, com partículas primárias da ordem de $100 \mathrm{~nm}$, que formaram agregados aparentemente menores que os demais solventes.

\section{BIBLIOGRAFIA}

[1] JAYMES, I., DOUY, A., MASSIOT, D., et al.,. "Characterization of mono and diphasic mullite precursor powders prepared by aqueous routes, $\mathrm{Al}$ and Si MAS-NMR spectroscopy investigations", Journal of Materials Science, v. 31, n. 18, pp. 4581-4589, Set. 1996.

[2] SCHNEIDER, H., SCHREUER, J., HILDMANN, B. "Structure and properties of mullite- A review", Journal of the European Ceramic Society, v. 28, n.2, pp. 329-344, 2008.

[3] ASGHARI, M., MOHAMMADI, T., AZIZNIA, A., et al., "Preparation and characterization of a thin continuous faujasite membrane on tubular porous mullite support", Desalination, v. 220, n.1-3, pp. 65-71, Mar. 2008.

[4] ESHARGHAWI, A., PENOT, C., NARDOU, F. "Contribution to porous mullite synthesis from clays by adding $\mathrm{Al}$ and Mg powders", Journal of the European Ceramic Society, v. 29, n.1, pp. 31-38, Jan. 2009.

[5] CHEN, N., WANG, H., HUO, J., et al., "Preparation and properties of in situ mullite whiskers reinforced aluminum chromium phosphate wave transparent ceramics", Journal of the European Ceramic Society, v. 37, n. 15, pp. 4793-4799, Dec. 2017.

[6] LI, J., WANG, W., DU, H., et al., "Kaolin/silica/mullite fiber multilayer membranes for high-efficiency gas filtration”, Ceramics International, v. 46, pp. 28742-28748, Set. 2020.

[7] CHEN, M., ZHU, L., DONG, Y., et al., "Waste-to-resource strategy to fabricate highly porous whiskerstructured mullite ceramic membrane for simulated oil-in-water emulsion wastewater treatment", ACS Sustainable Chemistry \& Engineering, v. 4, n.4, pp. 2098-2106, Feb. 2016.

[8] WANG, Y., LIU, J. “Aluminum phosphate-mullite composites for high-temperature radome applications", International Journal of Applied Ceramic Technology, v. 6, n. 2, pp. 190-194, Mar. 2009.

[9] PYZIK, A.J., TODD, C.S., HAN, C. "Formation mechanism and microstructure development in acicular mullite ceramics fabricated by controlled decomposition of fluorotopaz", Journal of the European Ceramic Society, v. 28, n. 2, pp. 383-391, 2008.

[10] LIU, M., ZHU, Z., ZHANG, Z., et al., "Development of highly porous mullite whisker ceramic membranes for oilin-water separation and resource utilization of coal gangue" Separation and Purification Technology, v. 237, n.15, Apr. 2020.

[11] SILVA, V.J., ALMEIDA, E.P., GONÇALVES, W.P., et al., "Mineralogical and dielectric properties of mullite and cordierite ceramics produced using wastes", Ceramics International, v. 45, n. 4, pp. 4692-4699, Mar. 2019.

[12] CHEN, Y., LIU, G., GU, Q., et al., "Preparation of corundum-mullite refractories with lightweight, high strength and high thermal shock resistance", Materialia, v. 8, Dec. 2019.

[13] RAGHDI, A., HERAIZ, M., SAHNOUNE, F., et al., "Mullite-zirconia composites prepared from halloysite reaction sintered with boehmite and zirconia", Applied Clay Science, v. 146, n. 15, pp. 70-80, Set. 2017.

[14] CHAUDHURI, S.P., PATRA, S.K. "Preparation e characterization of transition metal ion doped mullite", Transactions and Journal of the British Ceramic Society, v. 97, n. 3, pp.105-111, 1997. 
[15] ERTUGRUL, O., DALMIS, R., AKPINAR, S., et al., "Influence of zircon particle size on conventional and microwave assisted reaction sintering of in-situ mullite-zirconia composites", Ceramics International, v. 42, n. 9, pp. 11104-11117, Jul. 2016.

[16] BRAGA, A.N.S., SIMÕES, V.N., LIRA, H.L., et al., "Influência do tipo de solvente na síntese de mulita pelo método Pechini", Cerâmica, v. 65, n. 375, pp. 388-393, 2019.

[17] LIU, D., TAN, M., FANG, C., et al., "Mullite fiber cloth-reinforced mullite composite fabricated via an optimized layer-by-layer assembly method", Ceramics International, v. 45, n. 1, pp. 304-311, Jan. 2019.

[18] GHANE, A. S., AGHAJANI, H. "In situ synthesis of mullite by electrophoretic deposition method for bio-related applications", Materials Today: Proceedings, v. 5, n. 7, pp. 15653-15660, 2018.

[19] YOSHIDA, K., HYUGA, H., KONDO, N., et al., "Synthesis of precursor for fibrous mullite powder by alkoxide hydrolysis method". Materials Science and Engineering B, v.173, n. 1-3, pp. 66-71, Oct. 2010.

[20] ZENG, D., ZHANG, H., YANG, J., et al., "Microstructure and property of porous mullites with a whiskers framework obtained by a sol-gel process", Ceramics International, v. 42, n. 9, pp. 11270-11274, Jul. 2016.

[21] KAKIHANA, M. "Invited review sol-gel preparation of high temperature superconducting oxides",Journal of sol-gel Science and Technology, v. 6, n.1, pp. 7-55, Jan. 1996.

[22] KAKIHANA, M., YOSHIMURA, M. "Synthesis and characteristic of complex multicomponent oxides prepared by polimer complex method", Bulletin of the Chemical Society of Japan, v. 72, n. 7, pp. 1427-1443, Jul. 1999.

[23] BHOSALE, R.R. "Synthesis and characterization of nanocrystalline $\mathrm{CoFe}_{2} \mathrm{O}_{4}$-zirconia via propylene oxide aided sol-gel method", Ceramics International, v. 44, n. 7, pp. 8679-8683, May. 2018.

[24] BAYAL, N., JEEVANANDAM, P. "Synthesis of $\mathrm{TiO}_{2}-\mathrm{MgO}$ mixed metal oxide nanoparticles via a sol-gel method and studies on their optical properties", Ceramics International, v. 40, n. 10, pp. 1546315477, Dec. 2014.

[25] JARMAN, R.H., BAFIA, J., GEBRESLASSE, T., et al., "Synthesis of the p-type semiconducting ternary oxide $\mathrm{CuAlO}_{2}$ using the Pechini method", Materials Research Bulletin, v.48, n. 10, pp. 3916-3918, Oct. 2013.

[26] BRAGA, A.N.S., FARIAS, R.M.C., COSTA, D.L., et al., "Synthesis of Mullite by the Pechini Method", Materials Science Forum, v. 820, pp. 107-112, Jun. 2015.

[27] CESCONETOA, F.R., BORLAFA, M., NIETO, M.I., et al., "Synthesis of $\mathrm{CaTiO}_{3}$ and $\mathrm{CaTiO}_{3} / \mathrm{TiO}_{2}$ nanoparticulate compounds through $\mathrm{Ca}^{2+} / \mathrm{TiO}_{2}$ colloidal sols: Structural and photocatalytic characterization", Ceramics International, v.44, n. 1, pp. 301-309, Jan. 2018.

[28] MANDIĆ, V., KURAJICA, S. "The influence of solvents on sol-gel derived calcium aluminate", Materials Science in Semiconductor Processing, v. 38, pp. 306-313, Oct. 2015.

[29] BADNORE, A.U., CHAUDHARI, A.P., PATEL, J.K., et al., "Effect of solvents on properties of the ultrasound assisted synthesized ceria nanoparticles and its performance as an adsorbent", Advanced Powder Technology, v. 30, n. 5, pp. 1058-1066, May. 2019.

[30] GU, H., SOUCEK, M.D. "Preparation and characterization of monodisperse cerium oxide nanoparticles in hydrocarbon solvents", Chemistry of Materials, v. 19, n. 5, pp. 1103-1110, Feb. 2007.

[31] SOLA, E.R., TORRES, F.J., ALARCON, J. "Thermal evolution and structural study of 2:1 mullite from monophasic gels”, Journal of the European Ceramic Society, v. 26, n. 12, pp. 2279-2284, 2006.

[32] AMUTHARANI, D., GNANAM, F.D. "Low temperature pressure less sintering of sol-gel derived mullite", Materials Science and Engineering, v. 264, n. 1-2, pp.254-261, May. 1999.

[33] ZHANG, G., FU, Z., WANG, Y., et al., "Boron-doped mullite derived from single-phase gels", Journal of the European Ceramic Society, v. 30, n. 12, pp.2435-2441, Set. 2010.

[34] KURAJICA, S., TKALCEC, E., MANDIC, V., et al., "Mullite crystallization kinetics of lanthanum doped sol-gel derived precursors", Journal of the European Ceramic Society, v.31, n. 3, pp.377-383, Mar. 2011.

[35] REN, L., FU, Z., WANG, Y., et al., "Effect of the addition of glucose on the fabrication of nanosized mullite from a sol-gel by spark plasma sintering", Journal of the European Ceramic Society. v. 35, n. 6, pp. 1915-1921, Jun. 2015. 
[36] HUANG, Y.X., SENOS, A.M.; ROCHA, J., et al., "Gel formation in mullite precursors obtained via tetraethylorthosilicate (TEOS) pré-hydrolysis”, Journal of Materials Science, v. 32, n. 1, pp.105-110, Jan. 1997.

[37] KANSAL, P., LAINE, R.M., BABONNEAU, F. “A processable mullite precursor prepared by reacting silica and aluminum hydroxide with triethanolamine in ethylene glycol: structural evolution on pyrolysis", Journal of the American Ceramic Society, v. 80, n. 10, pp. 2597-2606, Oct. 1997.

[38] LEIVO, J., LINDEN, M., ROSENHOLM, J.M., et al., "Evolution of aluminosilicate structure and mullite crystallization from homogeneous nanoparticulate sol-gel precursor with organic additives", Journal of the European Ceramic Society, v.28, n. 8, pp.1749-1762, 2008.

[39] GUO, X., LI, W., NAKANISHI, K., et al., "Preparation of mullite monoliths with well-defined macropores and mesostructured skeletons via the sol-gel process accompanied by phase separation", Journal of the European Ceramic Society, v.33, n. 10, pp. 1967-1974, Set. 2013.

[40] MORITZ, K., HERBIG, R., DAMJANOVI'C, T., et al., "Development of mullite and mullite/ $\mathrm{Al}_{2} \mathrm{O}_{3}$ precursor sols for electrophoretic deposition of oxidation protection coatings", Colloids and Surfaces A: Physicochemical and Engineering Aspects, v. 350, n. 1-3, pp. 13-21, Oct. 2009.

\section{ORCID}

Aluska do Nascimento Simões Braga

Geysivana Kessya Garcia Carvalho

Hélio de Lucena Lira

Gelmires de Araújo Neves

Romualdo Rodrigues Menezes https://orcid.org/0000-0002-7764-6147

https://orcid.org/0000-0001-7589-7148

https://orcid.org/0000-0002-1527-9935

https://orcid.org/0000-0002-2900-1600

https://orcid.org/0000-0003-4316-2168 